\title{
The readout of a GEM- or Micromegas-equipped TPC by means of the Medipix2 CMOS sensor as direct anode
}

\author{
P. Colas ${ }^{\text {a }}$, A.P. Colijn ${ }^{\text {b }}$, A. Fornaini ${ }^{\text {b }}$, Y. Giomataris ${ }^{\text {a }}$, \\ H. van der Graaf ${ }^{b}$, E.H.M Heijne ${ }^{c}$, X. Llopart ${ }^{c}$, J. Schmitz ${ }^{d}$, \\ J. Timmermans ${ }^{b}$, J.L. Visschers ${ }^{b}$ \\ ${ }^{\mathrm{a} D A P N I A, C E A}$ Saclay, 91191 Gif sur Yvette cedex, France \\ ${ }^{\mathrm{b}}$ Nationaal Instituut voor Kernfysica and Hoge-Energiefysica (NIKHEF), P.O. \\ Box 41882 - 1009DB Amsterdam, The Netherlands \\ ${ }^{\mathrm{c}}$ CERN/Medipix Consortium, Geneva, Switzerland \\ ${ }^{\mathrm{d}}$ Univ. of Twente/MESA+, The Netherlands
}

Corresponding Author: Harry van der Graaf, NIKHEF, vdgraaf@nikhef.nl

\begin{abstract}
We have applied the Medipix2 pixel CMOS chip as direct anode readout for a TPC. For the gas amplification two options have been investigated: (i) a threestage GEM system and (ii) a Micromegas mesh. The structure of the cloud of primary electrons, left after interactions of ${ }^{55} \mathrm{Fe}$ quanta with the gas is visible with unprecedented precision. This proof-of-principle is an essential step in our project to realize a monolithic pixel sensor with integrated Micromegas, to be developed specially for the readout of TPCs, and applicable for drift chambers in general.
\end{abstract}

Keywords : GEM, Micromegas, Medipix2, TPC, pixel, pixel segmented anode

\section{Introduction}

Our goal is to develop a single-electron sensitive monolithic device TimePixGrid consisting of a CMOS pixel matrix TimePix covered with a Micromegas [1]. Each pixel is equipped with a preamp, a discriminator, a threshold DAC and time stamp circuitry. Such a sensor would replace the 
wires (or GEMs, or Micromegas), anode pads, feedthrough, readout electronics and cables of TPCs and could generally be applied in gaseous (drift) chambers. We intend to fabricate the Micromegas grid onto the TimePix chip by means of wafer post-processing technology.

The aim of the research presented in this paper is to prove the feasibility of a TPC readout system based on a CMOS pixel sensor in combination with either GEM foils or Micromegas.

\section{A pixel sensor + GEM system}

We applied the Medipix2 chip [2], [3], [4] as experimental readout device. This monolithic CMOS chip contains a square matrix of $256 \times 256$ pixels, each with dimensions $55 \times 55 \mu^{2}$. Each pixel is equipped with a low-noise preamp, discriminator, threshold DACs, a 14-bit counter and communication logic. One edge of the chip has aluminum bonding pads. The outer dimensions of the chip are $16.12 \times 14.11 \mathrm{~mm}^{2}$. The Medipix2 chip has been designed for X-ray imaging applications. For that particular application, an X-ray semiconductor converter (i.e. Si or CdZnTe), in the form of a corresponding pixel matrix, is mounted onto the Medipix2 chip, by means of bump-bonding. The assembly of a Medipix2 CMOS chip and a X-ray semiconductor converter forms a complete $\mathrm{X}$-ray imaging device. For each pixel the number of absorbed X-ray quanta in a given acquisition time is counted, and the combined pixel content forms the X-ray image.

For a proof-of-principle, we combined a stack of three GEMs [5] with the Medipix2 chip (without the X-ray semiconductor converter), as depicted in fig. 1. A small TPC was made with a drift volume of $100 \times 100 \times 100 \mathrm{~mm}^{3}$. The electric field in the drift volume was created by electrodes, in the form of square wire loops, put at linearly decreasing potentials. Insulating pillars, placed outside the drift volume, supported the corners of the wire loops. The printed circuit board of the Medipix2 was mounted in an insert in the aluminum base plate of the chamber. The surface of the Medipix2 was flush with the top plane of the base plate, in order to create an homogeneous drift field between the bottom GEM and the Medipix2.

All potentials were derived from a single resistor chain; the effective gas multiplication of the triple GEM could be varied by changing the potential of the top of the drifter. The dimensions and potentials of all the electrodes are listed in table 1.

The readout of our system is organized in the following way. The Medipix2 chip is connected through a Muros2 interface [4] to a PC equipped with a 
commercial NI PCI board. No triggering system was implemented: our readout was collecting all the ionization charge during a fixed acquisition time.

We irradiated the chamber with X-rays from ${ }^{55} \mathrm{Fe}$ and $\beta$ 's from ${ }^{90} \mathrm{Sr}$, using an Ar/Methane gas mixture. On April 1, 2003, we recorded the image of fig. 2. The signal response of the ${ }^{55} \mathrm{Fe}$ quanta was distributed over more pixels than expected, and a gain of $13 \mathrm{k}$ was required for the local pixels to cross their thresholds. We repeated these measurements in February 2004 with Ar/Isobutane 95/5, allowing an even higher gas gain (18 k), and an image is shown in fig. 3. Here, too, the distribution of the charge cloud ending on the pixel segmented anode is quite wide. Most of the ${ }^{55} \mathrm{Fe}$ quanta will be absorbed in the top section of the drift space, and the cloud of primary electrons will be widened due to diffusion during the drift over a large section of the $100 \mathrm{~mm}$ long drift length. This, in combination with de-focusing effects in GEMs, seen by other groups [6], may explain the images. Another explanation may be the too low extracting field below the GEMs.

We note that Bellazzini et al. [7] have applied a single GEM in combination with a direct pixel-segmented anode, where strong de-focusing was not seen. In his set-up, diffusion of primary and secondary electrons was limited to a minimum by a small distance between the GEM and the anode, and a small drift space.

\section{A pixel sensor + Micromegas system}

In a second step, we placed a Micromegas onto the Medipix2. The Micromegas itself is a copper foil, thickness $5 \mu \mathrm{m}$, with holes of $30 \mu \mathrm{m}$ diameter in a square pattern with $60 \mu \mathrm{m}$ pitch. The Micromegas is fixed onto the Medipix2 by means of poly-imide pillars (height $50 \mu \mathrm{m}$, diameter $200 \mu \mathrm{m}$, pitch (square) $0.8 \mathrm{~mm})$.

Originally, each pixel of the Medipix2 chip is covered with an insulating passivation layer; the conductive pad (octagonal pads $25 \mu \mathrm{m}$ wide) is large enough to accommodate a bump bond sphere. The electric field in the gap between the Medipix2 and the Micromegas is in the order of $7 \mathrm{kV} / \mathrm{mm}$, and discharges may be expected when some 70 percent of the anode surface is covered with an insulating material.

For this reason, the Medipix2 chips were post-processed at wafer level in the MESA + clean room. The post-processing consisted of a deposition of a thin aluminum layer using lift-off lithography. This allows deposition of metal on the anode matrix without modification of the bond pads. The pixel pads were enlarged to reach a metal coverage of $80 \%$ in the anode plane (see fig. 4). 
Electrical tests showed that the pre-amplifier functionality was unaffected by this post-processing.

A cathode plane $15 \mathrm{~mm}$ above the Micromegas creates a drift gap. On the drift cathode, a voltage of $-600 \mathrm{~V}$ was applied and $-(250-350 \mathrm{~V})$ on the Micromegas. The Medipix2 pixel pads were at ground potential. We flushed the chamber with Ar/Isobutane 95/5.

The signals from ${ }^{55} \mathrm{Fe}$ became visible at a Micromegas potential of $290 \mathrm{~V}$. We measured a gas multiplication factor of 300 for this voltage. Assuming an average number of 220 primary electrons released by most interacting ${ }^{55} \mathrm{Fe}$ quanta, about 66000 electrons will enter the anode plane. With a pixel discriminator threshold of about 3000 electrons, this signal can be detected even if the initial electron cloud is spread over an area of about 20 pixels.

When raising the $\mathrm{HV}$ of the Micromegas to $350 \mathrm{~V}$, micro-discharges at or near the Micromegas pillars became apparent (see fig. 5). The current due to these discharges was less than $0.5 \mathrm{nA}$ and could not be measured. The discharges decreased rapidly in amplitude and frequency. During operation, we never experienced a measurable excess current. Fig. 6 shows the image taken after strong irradiation with $\beta$ 's from a ${ }^{90} \mathrm{Sr}$ source. The dead regions caused by the pillars is clearly visible. Fig. 7 shows some ${ }^{55} \mathrm{Fe}$ events with an enlarged view of one of them. As is already visible from these few events, a readout system based on CMOS pixel sensor offers the unique possibility to directly view the processes involved in the absorption of X-ray quanta. In a more developed version of detectors based on this technology it is expected that for example Compton scattering, the photoelectric effect and the emission of Auger or $\delta$ electrons can be fully disentangled on an event-by-event basis [8].

\section{Conclusions}

We have successfully operated the Medipix CMOS pixel sensor as a direct anode. In a first set up the primary electrons originating from ${ }^{55} \mathrm{Fe}$ quanta absorbed in the drift gas, were multiplied by means of a set of three GEM foils. Probably due to the long drift path and the large distance between the last GEM and the Medipix2, and possibly due to de-focusing effects, diffuse images of X-ray conversions and charged particle tracks were recorded. In a second setup, we combined the Medipix2 with a Micromegas, and sharp images of ${ }^{55} \mathrm{Fe}$ events were observed. Apparently, the CMOS pixel sensor can withstand a strong electric field at its surface. This opens the possibility for a new readout technology of gaseous (drift) chambers. 


\section{Acknowledgment}

We thank the Medipix Collaboration for providing us with several wafers with Medipix2 chips, for the readout software and hardware, and for valuable advice. We would like to thank Arnaud Giganon, Wim Gotink and Joop Rövekamp for their creative and essential contributions to the realization of the test detectors. We thank Tom Aarnink, Ad Berkien, Edward Berbee, Frank de Zwart, Dimitri John, Jan Martis and Jan van Veen for their contributions.

\section{References}

[1] Y. Giomataris et al.: Micromegas: a high-granularity position-sensitive gaseous detector for high particle-flux environments, Nucl. Instr. and Methods A 376 (1996) 29-35

[2] X. Llopart, M. Campbell, R. Dinapoli, D. San Segundo and E. Pernigotti: Medipix2, a $64 \mathrm{k}$ pixel read-out with $55 \mu \mathrm{m}$ square elements working in single photon counting mode, IEEE Trans. Nucl. Sci. 49 2279-83 (2002)

[3] M. Conti, M. Maiorino, G. Mettivier, M. C. Montesi, P. Russo: Preliminary test of Medisoft 4: control software for the Medipix2 readout chip, IEEE Trans. Nucl Sci. vol. 50, No. 4, 869-877 (2003)

[4] D. San Segundo Bello, M. van Beuzekom, P. Jansweijer, H. Verkooijen, J.L. Visschers, An interface board for the control and data acquisition of the Medipix2 chip, Nucl. Instr. and Methods A 509 (2003) 164-170

[5] F. Sauli et. al.: The gas electron multiplier (GEM), Nucl. Instr. and Methods A 386 (1997) 531

[6] D. Karlen et al.: Investigation of GEM Space Point Resolution for a TPC Tracker, Proceedings of the Linear Collider Workshop 2000, FNAL, USA http://www-lc.fnal.gov/proceedings/d3_vertex/Dean_Karlen.pdf

[7] R. Bellazzini: Proceedings of the Xth Vienna conference on instrumentation, 2004, arXiv:physics/0403019.

[8] M. Hauschild, Proceedings of the International Workshop on Linear Colliders 2002, Jeju, Korea, J.S. Kang and S.K. Oh (editors), 464-469 


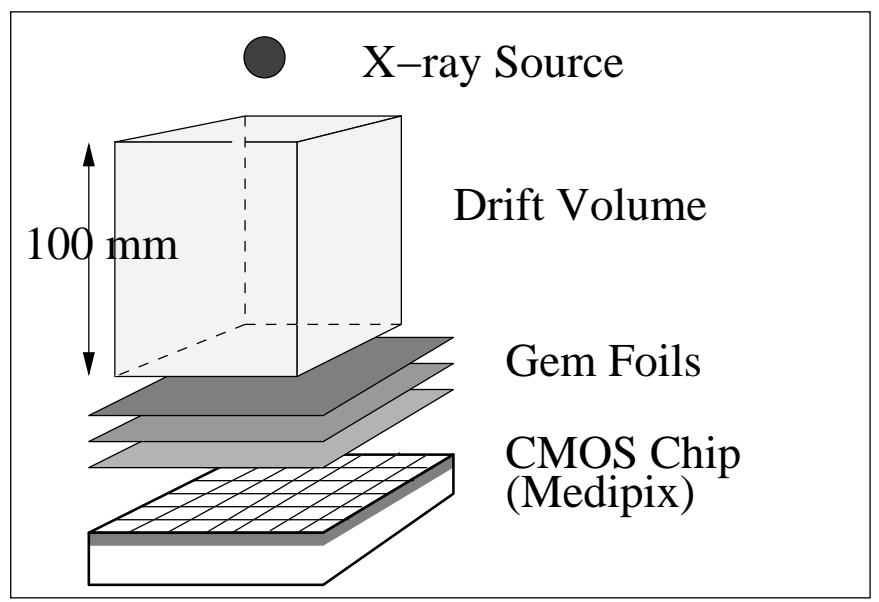

Fig. 1. The layout of the drift space, triple GEM and Medipix2 in the prototype test TPC.

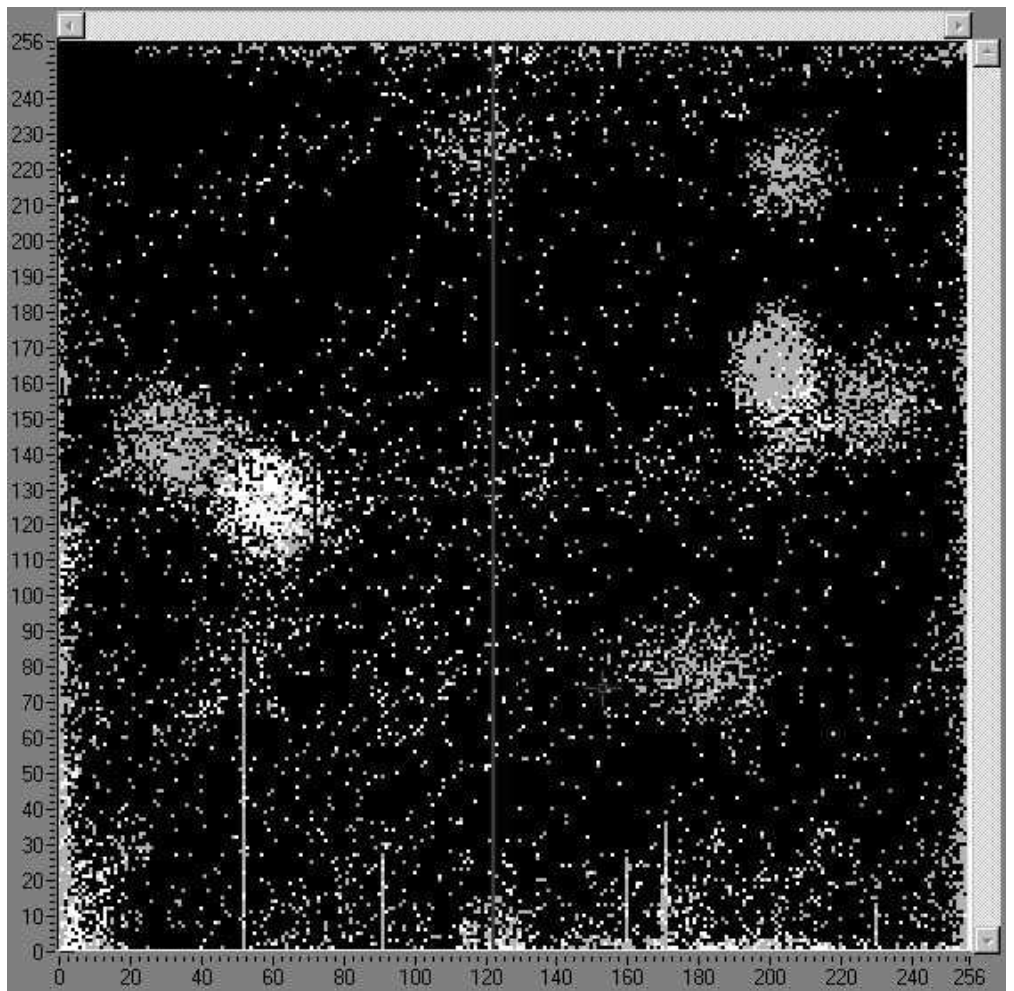

Fig. 2. Image recorded from the Medipix2 sensor placed below the triple GEM: all the ionization charge during an acquisition time of $1 \mathrm{sec}$. is recorded. An ${ }^{55} \mathrm{Fe}$ source was placed close to the chamber. The gas employed is Ar/Methane 90/10, the gas gain is $13 \mathrm{k}$. The resulting charge distribution is wider than expected. Color scheme: black $=0$ hits above threshold, grey $=1$ hit, white $=2$ hits during the acquisition time window. 


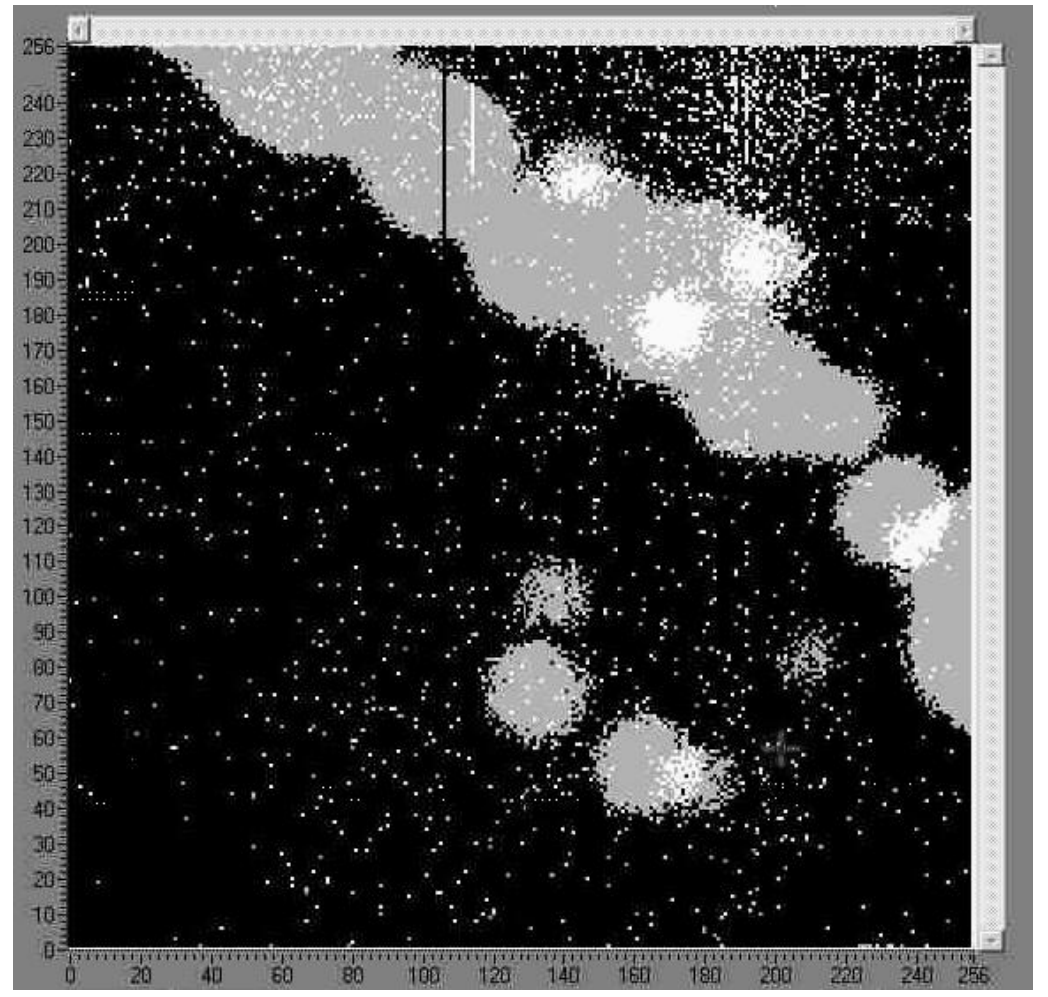

Fig. 3. As fig. 2, but with $\mathrm{Ar} /$ Isobutane $95 / 5$, gain $18 \mathrm{k}$, no radioactive source present and a 2 sec. acquisition time. A non-typical image of cosmic ray track is visible.

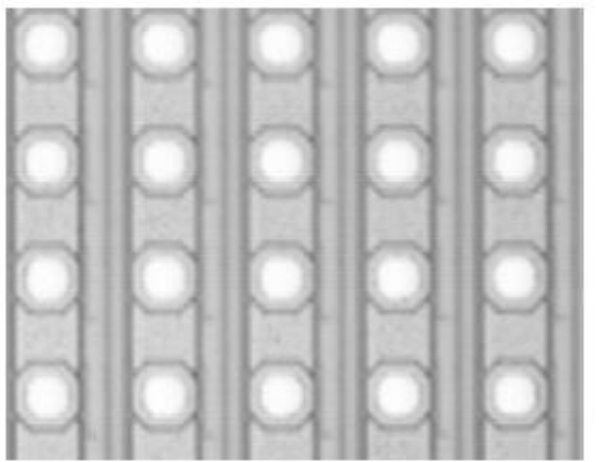

a)

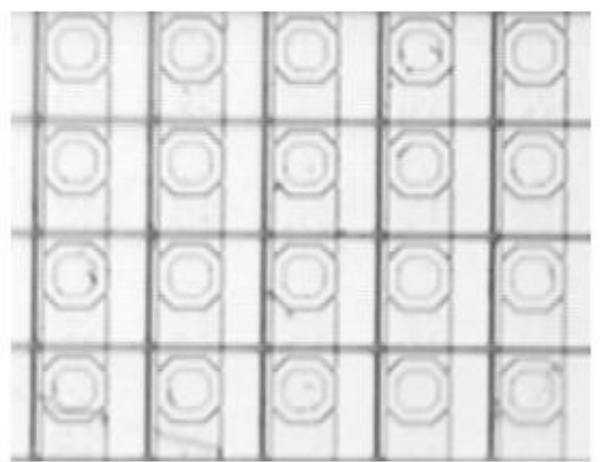

b)

Fig. 4. The Medipix2 chip before (a) and after (b) the wafer post processing. The original pad $(\mathrm{Al}, 25 \times 25 \mu \mathrm{m})$ is covered with an aluminum pad of $45 \times 45 \mu \mathrm{m}$. Note that no Micromegas mesh has yet been mounted on the chips shown in this figure. 


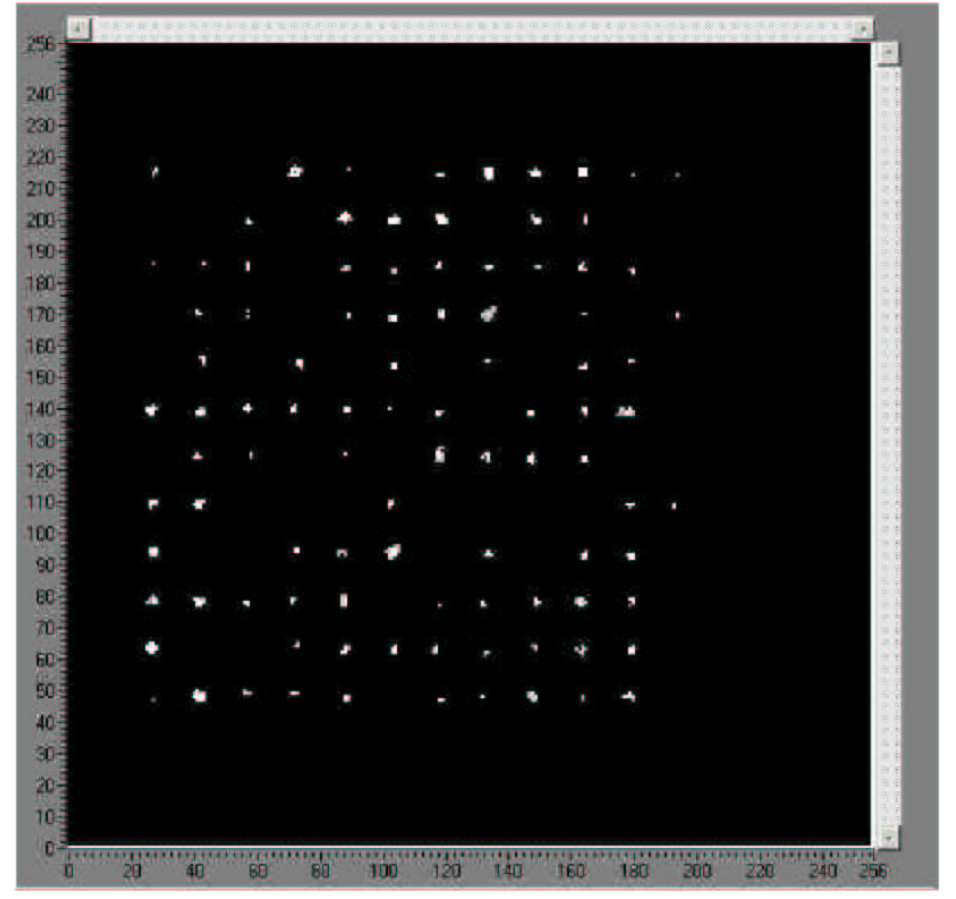

Fig. 5. Image acquired with the Medipix2/Micromegas prototype TPC. The measured gas multiplication factor is 1200 . Discharges near the pillars are apparent.

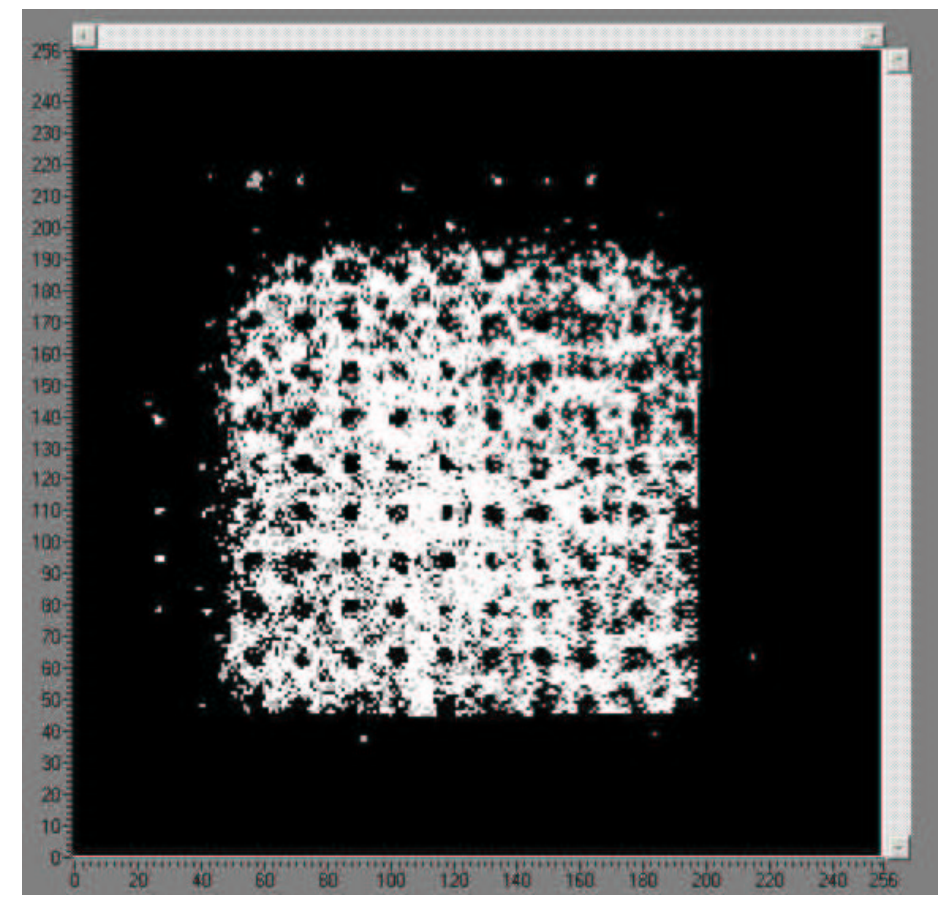

Fig. 6. Image acquired with the Medipix2/Micromegas prototype TPC. Strong irradiation with ${ }^{90} \mathrm{Sr}$. The shadows of the pillars are clearly visible. 


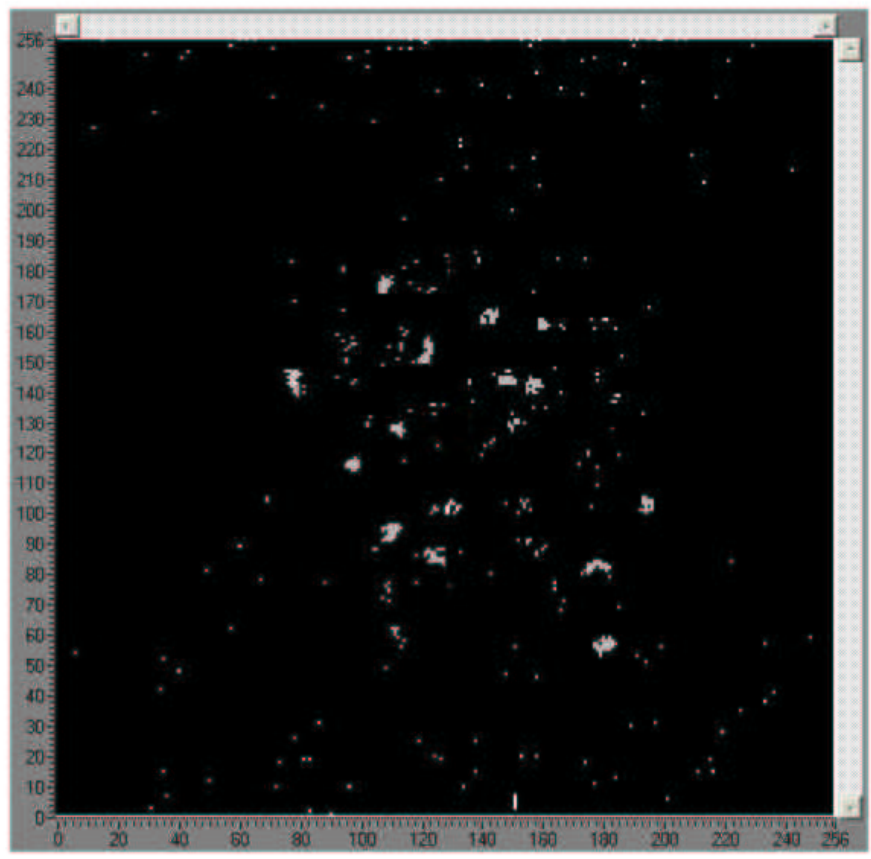

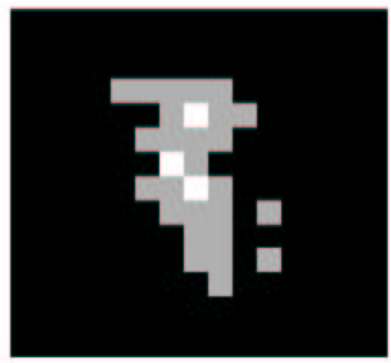

b)

a)

Fig. 7. a) Image acquired with the Medipix2/Micromegas prototype TPC. b) Enlarged view of an ${ }^{55} \mathrm{Fe}$ event from a): this structure results from detecting two clusters of charge, one originating from a fluorescence electron and the other from an Auger electron.

\begin{tabular}{|c|c|c|c|c|c|c|}
\hline \multirow{2}{*}{$\begin{array}{c}\text { MEDIPIX2 } \\
+ \\
\text { Triple GEM }\end{array}$} & \multicolumn{3}{|c|}{ April 1, 2003} & \multicolumn{3}{|c|}{ February 9, 2004} \\
\hline & $\begin{array}{l}\text { Distance to } \\
\text { Medipix2 } \\
(\mathrm{mm})\end{array}$ & \multicolumn{2}{|c|}{ Potential (-V) } & $\begin{array}{l}\text { Distance to } \\
\text { Medipix2 } \\
(\mathrm{mm})\end{array}$ & \multicolumn{2}{|c|}{ Potential (-V) } \\
\hline Top Drifter & 117.2 & \multicolumn{2}{|c|}{5580} & 118.0 & \multicolumn{2}{|c|}{5400} \\
\hline Bottom Drifter & 17.2 & \multicolumn{2}{|c|}{3355} & 18.0 & \multicolumn{2}{|c|}{3560} \\
\hline \multirow[t]{2}{*}{ GEM 1} & \multirow[t]{2}{*}{9.8} & Top & 2770 & \multirow[t]{2}{*}{10.6} & Tор & 3075 \\
\hline & & Bottom & 2420 & & Bottom & 2785 \\
\hline \multirow{2}{*}{ GEM 2} & \multirow[t]{2}{*}{8.2} & Top & 1830 & \multirow[t]{2}{*}{8.6} & Tор & 2300 \\
\hline & & Bottom & 1480 & & Bottom & 2010 \\
\hline \multirow[t]{2}{*}{ GEM 3} & \multirow[t]{2}{*}{6.6} & Top & 893 & \multirow[t]{2}{*}{6.6} & Top & 1525 \\
\hline & & Bottom & 541 & & Bottom & 1230 \\
\hline Gas Gain & \multicolumn{3}{|c|}{$13 \mathrm{k}$} & \multicolumn{3}{|c|}{$18 \mathrm{k}$} \\
\hline Gas mixture & Ar/Methane & \multicolumn{2}{|c|}{$90 / 10$} & Ar/Isobutan & \multicolumn{2}{|c|}{$95 / 5$} \\
\hline
\end{tabular}

Fig. 8. Table 1. Parameters of the triple GEM/Medipix2 setup. 